\title{
FRAUEN-
}

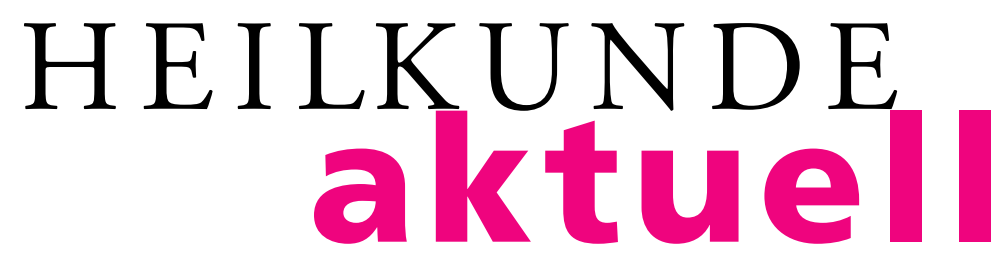

\section{Inhalt 11/1/2002}

Seite

Betrifft

Thema

Für Sie kommentiert

Wussten Sie schon...
«Name ist Schall und Rauch»?

Herausgeber

Adnextumore in der Schwangerschaft

Prof. Michael K. Hohl

Adjuvante Chemotherapie des Mammakarzinoms kann zu raschem

Knochenverlust führen / Prophylaktische Oxytocingabe zur Reduktion der postpartalen Blutung / Oxytocin oder Misoprostol (Cytotec $\left.{ }^{\circledR}\right)$ zur Prophylaxe der postpartalen Atonie?

Das Eintrittsalter in die Menopause ist weitgehend genetisch bestimmt / Der emotionale Stress der Patientinnen zu Beginn einer ART-Behandlung (IVF und GIFT) beeinflusste das Resultat negativ / Frauen mit verminderter Ovarialreserve (basales FSH > 14,2 IE/l) weisen eine extrem hohe Abortrate auf / Eine Tubenligatur bei Frauen mit $\mathrm{BrCA}_{1}$ - oder $\mathrm{BrCA}_{2}$-Mutation reduziert das Ovarialkarzinomrisiko deutlich / Orale Ovulationshemmer der 3. Generation haben ein deutlich geringeres Myokardinfarktrisiko als die der 2. Generation / Das Mortalitätsrisiko angegurteter Personen auf den Vordersitzen erhöht sich bei einem Autounfall durch nicht angegurtete Mitfahrer auf den Rücksitzen

Leseempfehlungen

Lesenswert

Wirtschaftlichkeitsuntersuchung

Forum

Mammakarzinom: «To screen or not to screen?»

oder: Ein Dilemma der sogenannten «evidence-based medicine»?

Prof. Bernhard Schüssler

Internet-News

www.google.de; www.nichd.nih.gov; www.ama-assn.org

Sertoli-Zelltumor

Der besondere Fall

Dr. Karin Schulz-Bischoff, Dr. Martin Frank,

Dr. Lutz Rathmer, Prof. Peter Scheidel

Feedback

Leserzuschrift

Dr. Hans Ikenberg 


\section{Wahlsectio \\ Obstet Gynecol Surv 2002;56:593-595}

$\beta$-Bockade bei schweren Verbrennungen zur Minderung des Katabolismus

N Engl J Med 2001;345:1223-1229

\section{Abwarten während der Austreibungsperiode}

Obstet Gynecol 2002;99:29-34

\section{out}

Suprazervikale endoskopische Hysterektomie Br J Obstet Gynaecol 2001;108:1017-1020

Hohe Sectiofrequenz bei Privatpatientinnen in Brasilien basiert auf Patientinnenwunsch

BMJ 2001;323:1155-1158

Synthetische oder natürliche Progesterone als effektive Behandlung des prämenstruellen Syndroms BMJ 2001;323:776-780
Impressum

\section{FRAUEN- \\ HEILKUNDE aktuel.}

S. Karger

Verlag für Medizin und Naturwissenschaften

Basel • Freiburg Paris $\cdot$ London New York • New Delhi Bangkok · Singapore Tokyo $\cdot$ Sydney

\section{Herausgeber}

Prof. Dr. med. M.K. Hohl

Chefarzt Frauenklinik

Kantonsspital Baden

CH-5404 Baden

Tel. +410564863502

Fax +410564863509

E-Mail frauenklinik@ksb.ch

Homepage: www.frauenklinik.ch

Prof. Dr. med. P. Scheidel

Chefarzt Frauenklinik

Marienkrankenhaus

Alfredstrasse 9

D-22087 Hamburg

Tel. +4904025461602

Fax +4904025461600

E-Mail scheidel.gyn@marienkrankenhaus.org

Für den Inhalt ausserhalb des redaktionellen Teiles (insbesondere Anzeigen, Industrieinformationen, Pressezitate und Kongressinformationen) übernehmen Schriftleitung, Beirat und Verlag keine Gewähr.

Eine Markenbezeichnung kann warenzeichenrechtlich geschützt sein, auch wenn bei ihrer Verwendung in dieser Zeitschrift das Zeichen ${ }^{\circledR}$ oder ein anderer Hinweis auf etwa bestehende Schutzrechte fehlen sollten. Für Satzfehler, insbesondere bei Dosierungsangaben, wird keine Gewähr übernommen.

Die Zeitschrift sowie alle in ihr enthaltenen einzelnen Beiträge und Abbildungen sind urheberrechtlich geschützt. Jede Verwertung, die nicht ausdrücklich vom Urheberrechtsgesetz zugelassen ist, bedarf der vorherigen Zustimmung des Verlags. Das gilt insbesondere für Vervielfältigungen, Bearbeitungen, Übersetzungen, Mikroverfilmungen und die Einspeicherung und Verarbeitung in elektronischen Systemen.

(C) Copyright 2002 S. Karger AG, Postfach, CH-4009 Basel (Schweiz) ISSN 1021-5697

Abonnementspreis

Ein Jahresabonnement (Kalenderjahr) kostet CHF 72.- (plus Versandkosten)

Die Zeitschrift erscheint 4mal jährlich 